\title{
Visualization of one-dimensional diffusion and spontaneous segregation of hydrogen in single crystals of $\mathrm{VO}_{2}$
}

\author{
T Serkan Kasırga ${ }^{1,2}$, Jim M Coy ${ }^{1}$, Jae H Park ${ }^{1}$ and David H Cobden ${ }^{1}$ \\ ${ }^{1}$ Department of Physics, University of Washington, Seattle, WA 98195, USA \\ ${ }^{2}$ UNAM-Institute of Materials Science and Nanotechnology, Bilkent University, Ankara 06800, Turkey \\ E-mail: kasirga@unam.bilkent.edu.tr
}

Received 4 May 2016, revised 23 June 2016

Accepted for publication 27 June 2016

Published 25 July 2016

\begin{abstract}
Hydrogen intercalation in solids is common, complicated, and very difficult to monitor. In a new approach to the problem, we have studied the profile of hydrogen diffusion in single-crystal nanobeams and plates of $\mathrm{VO}_{2}$, exploiting the fact that hydrogen doping in this material leads to visible darkening near room temperature connected with the metal-insulator transition at $65^{\circ} \mathrm{C}$. We observe hydrogen diffusion along the rutile $c$-axis but not perpendicular to it, making this a highly one-dimensional diffusion system. We obtain an activated diffusion coefficient, $\sim 0.01 \mathrm{e}^{-0.6 \mathrm{eV} / k_{\mathrm{B}} T} \mathrm{~cm}^{2} \mathrm{~s}^{-1}$, applicable in metallic phase. In addition, we observe dramatic supercooling of the hydrogen-induced metallic phase and spontaneous segregation of the hydrogen into stripes implying that the diffusion process is highly nonlinear, even in the absence of defects. Similar complications may occur in hydrogen motion in other materials but are not revealed by conventional measurement techniques.
\end{abstract}

S Online supplementary data available from stacks.iop.org/NANO/27/345708/mmedia

Keywords: vanadium dioxide, hydrogen doping, metal-insulator transition, 1D diffusion, optical microscopy

(Some figures may appear in colour only in the online journal)

Hydrogen dissolves in many solids, with effects on their physical properties which are relevant to storage [1], catalysis, sensing [2], and material degradation [3]. Understanding how hydrogen moves within a solid is thus important, but is very challenging because of the combination of the difficulty of detecting hydrogen, the complexity of the transport processes, and the sensitivity of kinetics and energetics to microscopic nonuniformity and defects [4]. It has traditionally been studied, predominantly in metals and semiconductors, by neutron scattering [5], nuclear [6] and muon magnetic resonance [7], and other techniques which do not yield spatial profiles. It is thus hard to know when deviations from a simple Fick's law behavior are relevant, such as when diffusion is guided by dislocations, grain boundaries or strain fields associated with twinning or structural modifications, or if there is spontaneous segregation. Recently, information on the spatial profile has been obtained using the change in color on hydrogenation of thin yttrium [8] and vanadium layers [9]. In these cases the behavior was explained using Fick's law, though some anomalies were present.

Hydrogen can also diffuse readily in some oxides [10], including rutile $\mathrm{TiO}_{2}$ and $\mathrm{VO}_{2}$ [11]. The rutile structure contains oxygen-lined channels parallel to the tetragonal $c$ axis along which hydrogen can hop between temporary $\mathrm{O}-\mathrm{H}$ bonds (figure 1(a)). Diffusion perpendicular to these channels requires the protons to move between densely spaced metal ions, and has a much higher energy barrier [12, 13]. Pure, unstrained rutile-structured $(\mathrm{R}) \mathrm{VO}_{2}$ has a well-known firstorder metal-insulator transition (MIT) to a monoclinic insulating phase (M1) on cooling through $T_{c}=65{ }^{\circ} \mathrm{C}[14,15]$. Interestingly, it has recently been found $[16,17]$ that hydrogen in $\mathrm{VO}_{2}$ reduces the gap in the insulating phase while 
(a)

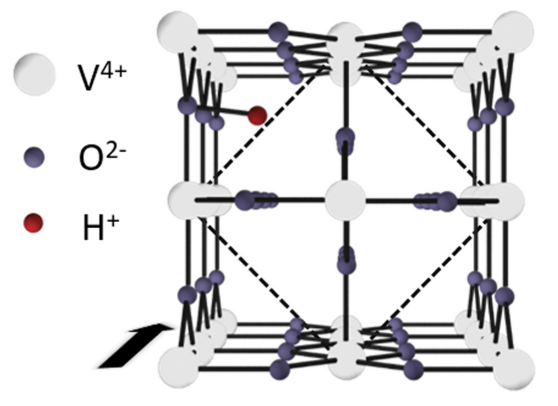

(c)

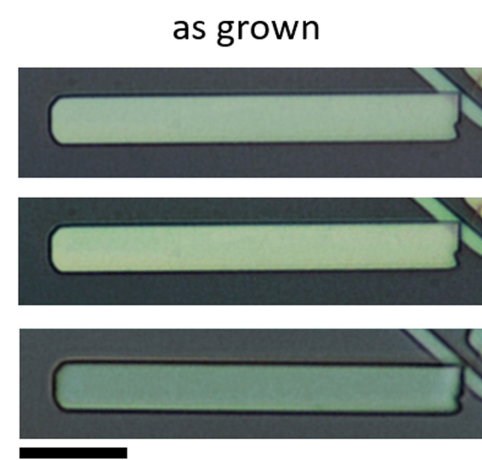

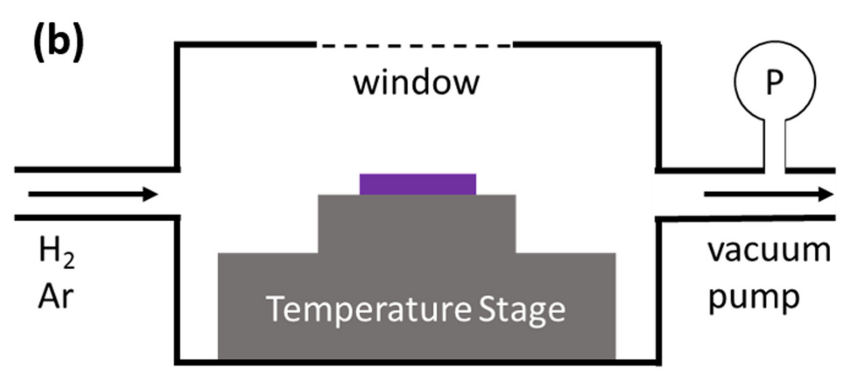

after $\mathrm{H}_{2}$ exposure

$23^{\circ} \mathrm{C}$

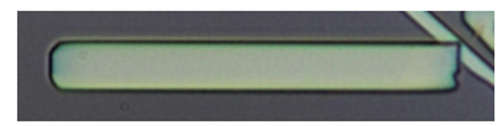

$60{ }^{\circ} \mathrm{C}$

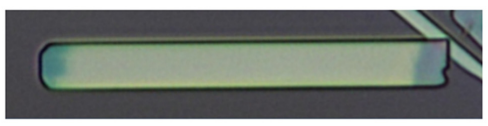

$70{ }^{\circ} \mathrm{C}$

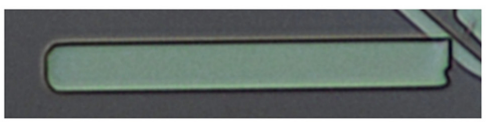

Figure 1. (a) View along the rutile $c$-axis shows the channels lined by oxygen atoms and a proton bound to one. Dashed line shows the base of the rutile unit cell and the black arrow indicates the direction of the rutile $c$-axis. (b) Schematic of the hydrogenation chamber, hermetically sealed with gas inlets and pump outlet. Gas flow is controlled by mass flow controllers and the chamber pressure is monitored by pressure sensors. The stage can be varied from $-40{ }^{\circ} \mathrm{C}$ to $200^{\circ} \mathrm{C}$. (c) Optical images of a $\mathrm{VO}_{2}$ nanobeam of thickness about $100 \mathrm{~nm}$, before and after hydrogen exposure (15 min of $5 \mathrm{ml} \mathrm{min}{ }^{-1} \mathrm{H}_{2}$ and $50 \mathrm{ml} \mathrm{min}^{-1} \mathrm{Ar}$ at $50 \mathrm{mBar}$ at $100^{\circ} \mathrm{C}$ ). Scale bar is $10 \mu \mathrm{m}$.

slightly reducing $T_{c}$, and the material becomes fully metallic above some concentration. Consequently, a thin $\mathrm{VO}_{2}$ sheet on a suitable substrate appears darker when hydrogenated, making it possible to directly image hydrogen motion in this oxide. In addition, small $\mathrm{VO}_{2}$ nanobeams and plates offer the unusual opportunity to study diffusion in an unstrained, domain-free single crystal.

To exploit this idea we prepared strain-free $\mathrm{VO}_{2}$ crystals and observed their darkening on exposure to hydrogen gas, using a palladium catalyst to dissociate the hydrogen on the surface. We thereby successfully determined the diffusion coefficient along the $c$-axis and its activation energy. We found that it is orders of magnitude greater than transverse to the channel axis, as in rutile $\mathrm{TiO}_{2}$, making the diffusion onedimensional. More surprisingly, we observed extreme supercooling of the metallic state, and spontaneous segregation of the hydrogen into stripes during diffusion. The latter implies that the diffusion process deviates strongly from Fick's law-a fact that would be disguised in conventional measurements. As a result, it appears impossible to achieve stable uniform moderate concentrations of hydrogen in $\mathrm{VO}_{2}$, as would be desirable for tuning the MIT for applications. We postulate that the segregation is related to the recently reported [18] structural change from monoclinic (M1) to orthorhombic ( $\mathrm{O} 1$ and $\mathrm{O} 2)$ occurring at moderate $\mathrm{H}$ doping levels, with elastic energies favoring a domain structure. Such segregation may be common in other solids (hydrogen uptake is typically accompanied by changes in the equilibrium structure) but has not been noticed because of the lack of probes.

We grow the crystals by physical vapor transport on oxidized silicon wafers [19]. They are usually elongated along the rutile $c$-axis, and when released from the substrate they are optically uniform and exhibit sharp MITs at $65^{\circ} \mathrm{C}$ implying a lack of impurities or twinning [15, 20, 21]. To counter strain caused by substrate adhesion [22], the crystals are either transferred to a soft polymer, polydimethylsiloxane (PDMS), or cantilevered from the edge of an oxidized silicon chip. To transfer the crystals on to PDMS, a PDMS coated chip is pressed against the growth substrate. We dip the substrate briefly dipped into buffered oxide etchant solution to loosen the crystals for a higher yield transfer and to remove any oxide formation on the surface of the crystals. $1 \mathrm{~nm}$ of $\mathrm{Pd}$ is evaporated to catalyze dissociation of $\mathrm{H}_{2}$ on the $\mathrm{VO}_{2}$ surface [23] (see supplementary material for further details). They are then exposed to hydrogen carried by argon flowing through a sealed chamber as indicated in figure 1(b).

Figure 1(c) shows optical images of a $\mathrm{VO}_{2}$ nanobeam on PDMS at several temperature, before (left) and after (right) an exposure to $\mathrm{H}_{2}$ at $100{ }^{\circ} \mathrm{C}$ (thus in the $\mathrm{R}$ phase). At $70{ }^{\circ} \mathrm{C}$, before exposure the nanobeam is fully metallic, and after exposure it looks exactly the same. At $23{ }^{\circ} \mathrm{C}$, before exposure it is fully insulating (paler color), and after exposure a faint darkening is visible near the ends. At $60{ }^{\circ} \mathrm{C}$, before exposure it is still fully insulating, but after exposure there are distinct regions at the ends having the full metallic darkness. We directly infer that hydrogen enters from at the ends, moving 
(a)

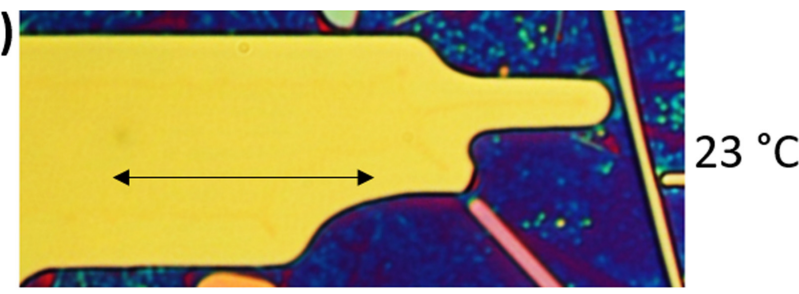

(b)
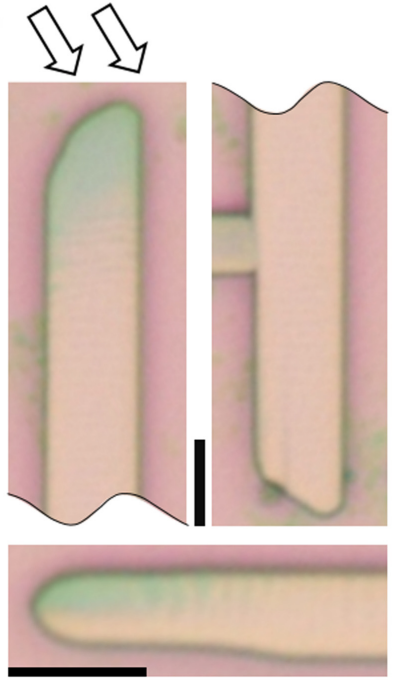

(c)

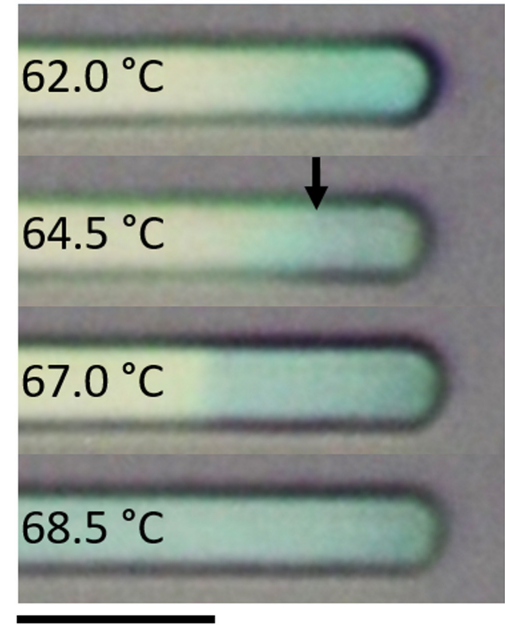

(d)

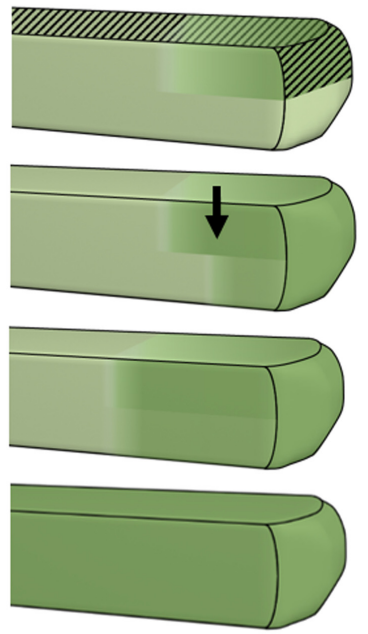

Figure 2. (a) Images of an irregularly shaped plate crystal at $23{ }^{\circ} \mathrm{C}$ before (left) and after (right) hydrogen exposure, clearly manifesting onedimensional diffusion along the channel axis (arrow). Scale bar is $10 \mu \mathrm{m}$. (b) Images of crystals with oblique Pd evaporation (direction indicated by the arrow) after hydrogen exposure. The top two images are the ends of the same nanobeam. Darkening only occurs from the coated edges, demonstrating the importance of the Pd catalyst for hydrogen injection. (c) Images of a nanobeam on PDMS after hydrogen exposure. The arrow indicates a boundary between two regions with different darkening. (d) Possible explanation for this. Hydrogen enters mainly the upper side of the end where Pd is present (indicated by hatching), leading to a lower concentration in the underneath side adjacent to the substrate. Scale bars are $5 \mu \mathrm{m}$.

along the $c$-axis on a scale of several microns and producing a concentration gradient so that both the insulator gap and $T_{c}$ are reduced near the ends. A lack of darkening far from the ends indicates that negligible hydrogen enters from the top surface, which is also covered with catalyst, in spite of the much shorter distance involved $(\sim 50 \mathrm{~nm})$ for diffusion perpendicular the $c$-axis.

The extreme anisotropy of the diffusion is manifested in many ways. For one example, in crystals with complicated shapes, such as the one shown in figure 2(a), the edge of the metallized region after exposure has the shape of the edge of the crystal translated along the $c$-axis. For another example, if we evaporate the $\mathrm{Pd}$ at a shallow oblique angle so that crystal side walls facing away from the source are not coated with $\mathrm{Pd}$, we do not see darkening near these sides (figure 2(b)). This also demonstrates the importance of the Pd catalyst.

Another common observation is illustrated in figure 2(c). In this nanobeam while warming we see distinct regions with different levels of darkening. This could be partly explained by a difference in color between doped insulator and the metallic phase, but another factor is in play. The end of a nanobeam is often pyramidal, and when the Pd is evaporated from above only the upper part of the end is covered, as sketched in figure 2(d). Hence hydrogen only enters the upper part of the crystal, which thus darkens further from the end at a given temperature.

Fortunately, some crystals on PDMS exhibited behavior consistent with uniform hydrogen injection at the ends at moderate exposure levels. Figure 3(a) shows a series of images of such a nanobeam after exposure to an $\mathrm{H}_{2}$ : $\mathrm{Ar}$ (1:10) gas mixture at $100{ }^{\circ} \mathrm{C}$ for different lengths of time $t$. They are taken at $60{ }^{\circ} \mathrm{C}$, because the darkening is greater than at room temperature yet the metal-insulator boundary is not as sharp as it is near $T_{C}$. We see a darkness gradient related to the local hydrogen concentration resembling a simple 1D diffusion profile. From Fick's law in one dimension, $\partial n / \partial t=D \partial^{2} n / \partial x^{2}$, with diffusion coefficient $D$, assuming zero concentration $n=0$ for time $t<0$ and a single boundary at $x=0$ at which $n$ is held at a constant value $n_{0}$ for $t>0$, we have $n(x, t)=n_{0} \operatorname{erfc}\left(\frac{x}{2 \sqrt{D t}}\right)$. According to this equation, after time $t$ the concentration decays by a half at distance $x=x_{m}$ where $x_{m} / 2 \sqrt{D t}=\operatorname{erfc}^{-1} 1 / 2 \approx 1 / 2$, or $x_{m}^{2} / t \approx D$.

To estimate $x_{m}$, the diffusion length, in the experiments we measure the positions at which the darkening is half that at the end, indicated by tick marks in figure 3(a). In figure 3(b) we plot $x_{m}^{2} / t$ for a series of exposure times $t$ at $100{ }^{\circ} \mathrm{C}$ (black). The results are consistent with the Fick's law prediction and yield $D=(8 \pm 1) \times 10^{-11} \mathrm{~cm}^{2} \mathrm{~s}^{-1}$. We estimate 
(a)

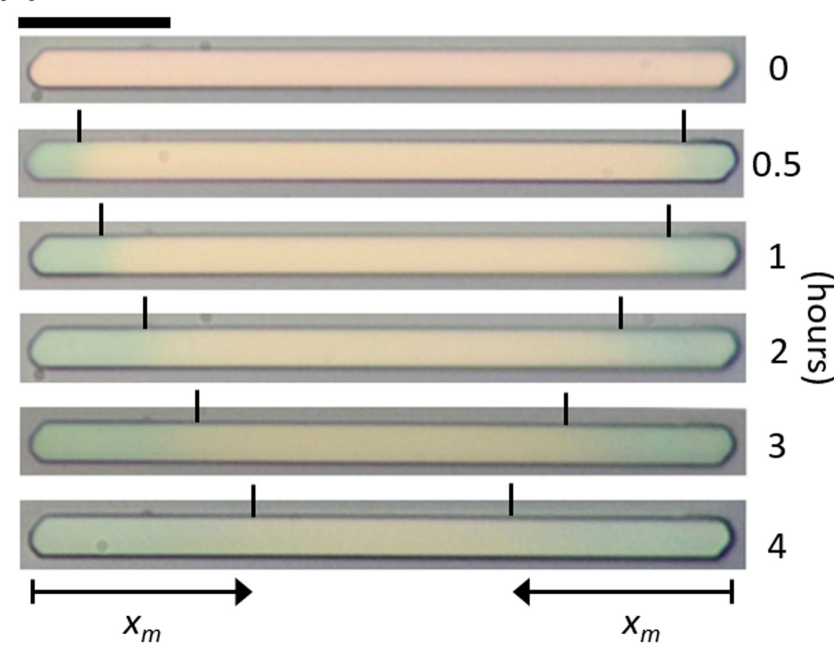

(b) $1000 / \mathrm{T}\left(\mathrm{K}^{-1}\right)$

(c)

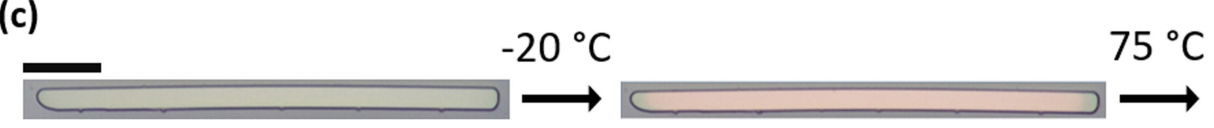

Figure 3. (a) Series of images taken at $60{ }^{\circ} \mathrm{C}$ before and after hydrogen exposure at $100{ }^{\circ} \mathrm{C}$ for the time $t$ indicated on the right. Ticks shows the position $x_{m}$ where the darkening is half that at the ends. All scale bars are $10 \mu \mathrm{m}$. (b) Plot for extracting the diffusion coefficient $D=x_{m}^{2} / t$ at several temperatures. The Arrhenius plot on the right of the graph yields activation energy $E_{a}=0.6 \mathrm{eV}$. (c) Supercooling seen at longer exposures $\left(2 \mathrm{~h}\right.$ at $\left.100{ }^{\circ} \mathrm{C}\right)$. All images are at room temperature.

the error here taking into account the fact that the darkness is an unknown nonlinear function of the hydrogen concentration. Even if there is for example a threshold value for hydrogen concentration to produce a color change, the effect on the estimated diffusion length will be minor since the temperature dependence of the position of the boundary is what matters. We also plot values obtained using other exposure temperatures $T$. An Arrhenius fit, shown on the right, yields an activation energy of $E_{a}=0.6 \pm 0.1 \mathrm{eV}$, similar to that reported for diffusion along the $c$-axis of $\mathrm{VO}_{2}$ [24] and $\mathrm{TiO}_{2}[12,13]$. Combining the results we find $D(T) \approx 0.01 \mathrm{e}^{-0.6 / k_{\mathrm{B}} T} \mathrm{~cm}^{2} \mathrm{~s}^{-1}$ along the rutile $c$-axis. These values are also consistent with the recent theoretical studies [25, 26].

At longer exposures (lower images in figure 3(a)), a complicating supercooling effect is present. Immediately after cooling from the fully metallic state (at $70^{\circ} \mathrm{C}$ ) to room temperature the crystals remain fully metallic. Only if left overnight, or cooled further, do they return to the insulating state with darkened ends as in the lower images in figure 3(a). After $4 \mathrm{~h}$ at $100{ }^{\circ} \mathrm{C}$ a dip in liquid nitrogen is needed to recover the insulating state. An example is shown in figure 3(c).

To eliminate any residual strain effects due to the PDMS substrate, we studied cantilevered nanobeams transferred using a micromanipulator onto the edge of an oxidized silicon chip and fixed with epoxy as sketched in figure 4(a). These were rinsed in dilute $\mathrm{HF}$ to remove surface $\mathrm{V}_{2} \mathrm{O}_{5}$ prior to evaporating $1 \mathrm{~nm}$ of $\mathrm{Pd}$ directed at the ends. Figure 4(b) shows three nanobeams of different size after hydrogen exposure. A metallic region can be seen with an interface at distance $x$ from the end. Figure 4(c) shows the variation of $x$ on cycling the temperature up (red) and down (blue). It is reproducible over two cycles, and almost identical for all three nanobeams, implying that this is intrinsic defect-free single-crystal behavior. In addition to the usual hysteresis in position of the large jump at the MIT, there is large hysteresis in the interface position implying that it is 'sticky' and its motion incurs a penalty of dissipated energy.

Most strikingly, our attempts to produce a uniform hydrogen concentration failed. After a long exposure $(4 \mathrm{~h}$ at $90{ }^{\circ} \mathrm{C}$ ), rather than seeing the darkening spread smoothly along the cantilevers we observed dark stripes which are faint but visible at room temperature, as shown in figure 4(d). On warming these become sharper, and then the paler regions between them turn dark too, but the insulating region beyond the last stripe does not darken until close to $65^{\circ} \mathrm{C}$ implying that it remains undoped. In addition, the period of the stripes increases, and each stripe propagates along the nanobeam and becomes darker, with increasing exposure time, as shown in figure 4(e).

The formation of stripes implies that the hydrogen tends to self-segregate in single-crystal $\mathrm{VO}_{2}$ (see supplementary materials for further evidence), and thus that there are large nonlinear diffusion forces that violate Fick's law. From the reproducibility between cantilevered nanobeams, the role of defects [4] and external strain [27] can be eliminated. Instead, the behavior can be explained qualitatively as follows: above a certain $\mathrm{H}$ concentration a structural distortion of the $\mathrm{R}$ phase becomes favorable to an orthorhombic $O$ phase [18] and $O$ domains appear with sizes that minimize the elastic energy cost of creating $\mathrm{O}-\mathrm{R}$ interfaces. Thereafter, hydrogen tends to 
(a)

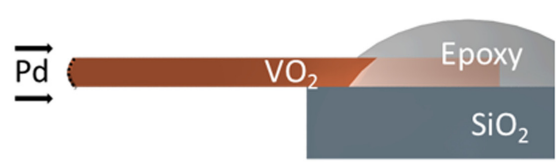

(b)
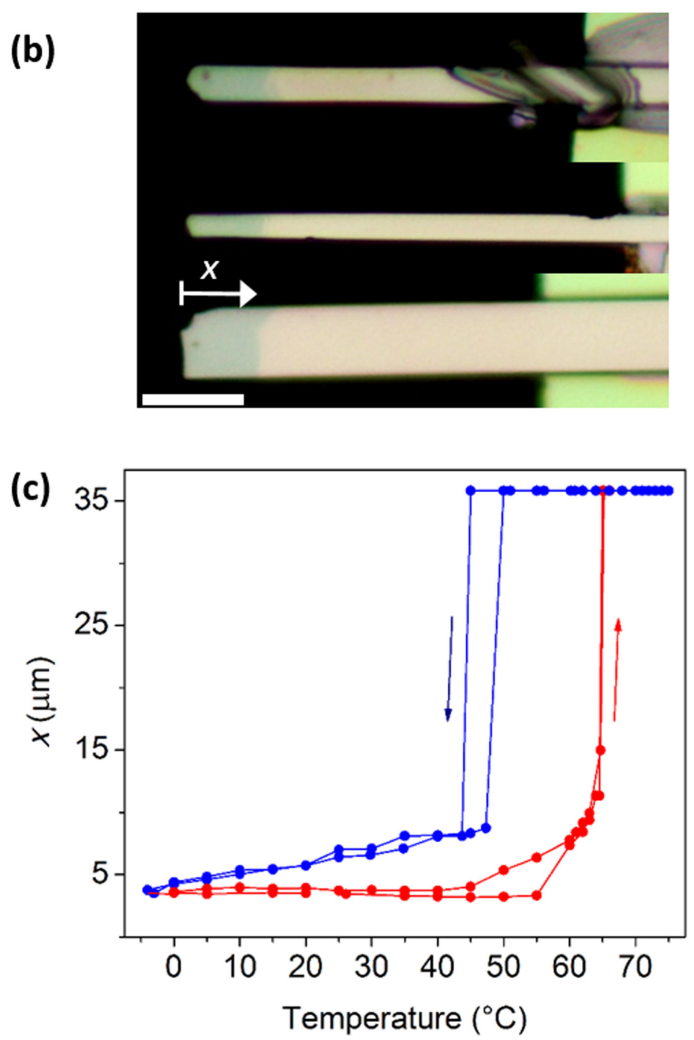

(d)

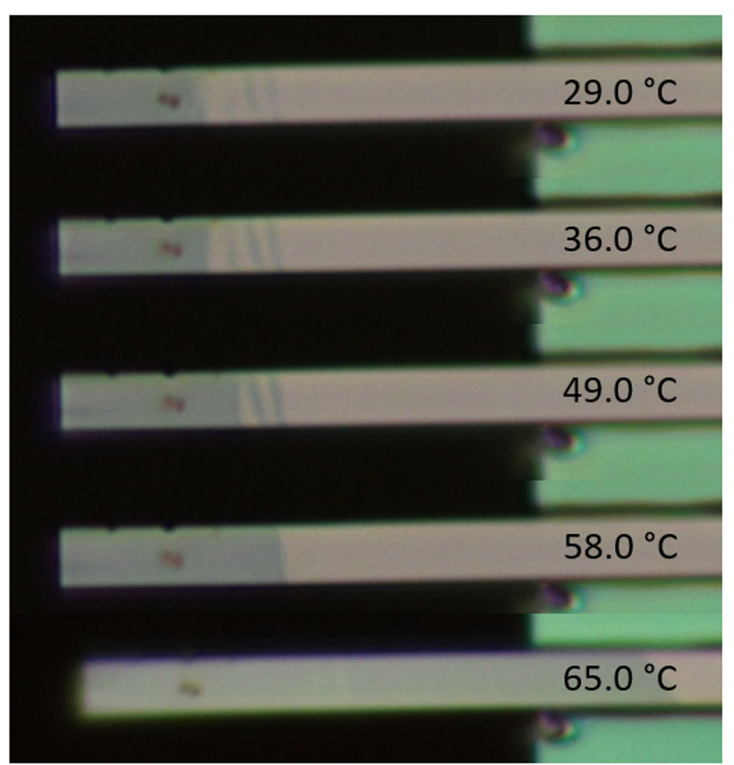

(e)

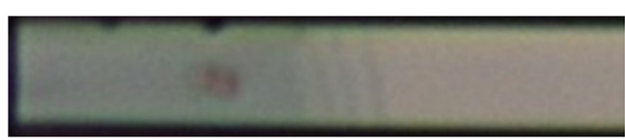

$3 \mathrm{~h}$

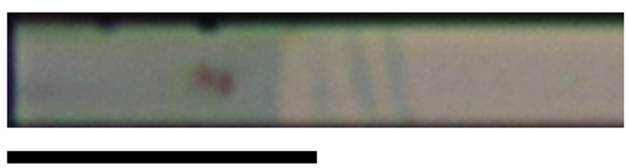

$4 \mathrm{~h}$

Figure 4. (a) Nanobeams are glued down cantilevered from the edge of a silicon chip and Pd catalyst is evaporated on the free ends. (b) Images at $55^{\circ} \mathrm{C}$ of three cantilevered nanobeams with different cross-sections after exposure to hydrogen for 20 min at $120^{\circ} \mathrm{C}$. All show the same metallized length $x$. (c) Measurement of $x$ versus temperature for one cantilever, cycled twice to the fully metallic state at $60{ }^{\circ} \mathrm{C}$. (d) Images at a series of temperatures of a cantilevered nanobeam after hydrogen exposure at $90{ }^{\circ} \mathrm{C}$ for $4 \mathrm{~h}$. Alternating darker and lighter stripes can be below $50{ }^{\circ} \mathrm{C}$. (e) The period of the stripes increases with exposure time. Images are taken at $36{ }^{\circ} \mathrm{C}$. Scale bars are all $10 \mu \mathrm{m}$.

move from $\mathrm{R}$ to $\mathrm{O}$ regions to further reduce the total free energy. Combined with the flow of hydrogen in from the free end this can produce evolving stripes of $\mathrm{O}$ phase. It seems likely that the stickiness of the metal-insulator interface and the supercooling described above are also related to the competition between different displacive structures combined with elastic energy and diffusion.

In summary, our studies of hydrogen diffusion in single crystals of $\mathrm{VO}_{2}$ by optical microscopy have revealed extreme anisotropy and highly nonlinear effects in diffusion that may be relevant in many other materials.

\section{Acknowledgments}

This work supported by the U.S. Department of Energy, Office of Basic Energy Sciences, Division of Materials Sciences and Engineering, Award DE-SC0002197 and Turkish Scientific and Technological Research Council (TUBITAK), Grant No 115F070.

\section{References}

[1] Schlapbach L and Züttel A 2001 Hydrogen-storage materials for mobile applications Nature $414353-8$

[2] Hübert T, Boon-Brett L, Black G and Banach U 2011 Hydrogen sensors- A review Sensors Actuators B 157 329-52

[3] Hirth J P 1980 Effects of hydrogen on the properties of iron and steel Metall. Trans. A 11 861-90

[4] Myers S M et al 1992 Hydrogen interactions with defects in crystalline solids Rev. Mod. Phys. 64559

[5] Rowe J M, Sköld K, Flotow H E and Rush J J 1971

Quasielastic neutron scattering by hydrogen in the $\alpha$ and $\beta$ phases of vanadium hydride J. Phys. Chem. Sol. 32 41-54

[6] Hofmann D M, Hofstaetter A, Leiter F, Zhou H, Henecker F, Meyer B K, Orlinskii S B, Schmidt J and Baranov P G 2002 Hydrogen: a relevant shallow donor in zinc oxide Phys. Rev. Lett. 88045504

[7] Kreitzman S R, Hitti B, Lichti R L, Estle T L and Chow K H 1995 Muon-spin-resonance study of muonium dynamics in Si and its relevance to hydrogen Phys. Rev. B $\mathbf{5 1} 13117$

[8] den Broeder F J A et al 1998 Visualization of hydrogen migration in solids using switchable mirrors Nature 394 $656-8$ 
[9] Palsson G K, Bliersbach A, Wolff M, Zamani A and Hjörvarsson B 2012 Using light transmission to watch hydrogen diffuse Nat. Commun 3892

[10] Fripiat J J and Lin X 1991 Hydrogen intercalation within transition metal oxides: entropy, enthalpy, and charge transfer J. Phys. Chem. 96 1437-44

[11] Chippindale A M, Dickens P G and Powell A V 1991 Synthesis, characterization, and inelastic neutron scattering study of hydrogen insertion compounds of $\mathrm{VO}_{2}$ (rutile) J. Solid State Chem. 93 526-33

[12] Johnson O W, Paek S-H and DeFord J W 1975 Diffusion of H and $\mathrm{D}$ in $\mathrm{TiO}_{2}$ : suppression of internal fields by isotope exchange J. Appl. Phys. 461026

[13] Bates J B, Wang J C and Perkins R A 1979 Mechanisms for hydrogen diffusion in $\mathrm{TiO}_{2}$ Phys. Rev. B 19 4130-9

[14] Morin F J 1959 Oxides which show a metal-to-insulator transition at the Neel temperature Phys. Rev. Lett. 334

[15] Park J H, Coy J M, Kasirga T S, Huang C, Fei Z, Hunter S and Cobden D H 2013 Measurement of a sold-state triple point at the metal-insulator transition in $\mathrm{VO}_{2}$ Nature $\mathbf{5 0 0}$ $431-4$

[16] Andreev V, Kapralova V and Klimov V 2007 Effect of hydrogenation on the metal-semiconductor phase transition in vanadium dioxide thin films Phys. Solid State $492318-22$

[17] Wei J, Ji H, Guo W H, Nevidomskyy A H and Natelson D 2012 Hydrogen stabilization of metallic vanadium dioxide in single-crystal nanobeams Nat. Nanotechnol. 7 357-62

[18] Filinchuk Y, Tumanov N A, Ban V, Ji H, Wei J, Swift M W, Nevidomskyy A H and Natelson D 2014 In situ diffraction study of catalytic hydrogenation of $\mathrm{VO}_{2}$ : stable phases and origins of metallicity J. Am. Chem. Soc. $1368100-9$

[19] Guiton B S, Gu Q, Prieto A L, Gudiksen M S and Park H 2005 Single-crystalline vanadium dioxide nanowires with rectangular cross sections J. Am. Chem. Soc. 127498

[20] Cao J et al 2010 Extended mapping and exploration of the vanadium dioxide stress-temperature phase diagram Nano Lett. 10 2667-73

[21] Wei J, Wang Z, Chen W and Cobden D H 2009 New aspects of the metal-insulator transition in single-domain vanadium dioxide nanobeams Nat. Nanotechnol. 4420

[22] Wu J, Gu Q, Guiton B S, de Leon N P, Ouyang L and Park H 2006 Strain-induced self organization of metal-insulator domains in single crystalline $\mathrm{VO}_{2}$ nanobeams Nano Lett. 6 2313-7

[23] Sermon P A and Bond G C 1973 Hydrogen spillover Catal. Rev. 8 211-39

[24] Lin J, Ji H, Swift M W, Hardy W J, Peng Z, Fan X, Nevidomskyy A H, Tour J M and Natelson D 2014 Hydrogen diffusion and stabilisation in single-crystal $\mathrm{VO}_{2}$ micro/nanobeams by direct atomic hydrogenation Nano Lett. 14 5445-51

[25] Warnick K H, Wang B and Pantelides S T 2014 Hydrogen dynamics and metallic phase stabilization in $\mathrm{VO}_{2} \mathrm{Appl}$. Phys. Lett. 104101913

[26] Cui Y, Shi S, Chen L, Luo H and Gao Y 2015 Hydrogendoping induced reduction in the phase transition temperature of $\mathrm{VO}_{2}$ : a first-principles study Phys. Chem. Chem. Phys. 17 20998-21004

[27] Gorsky W S 1935 Phys. Z. Sowjetunion 8457 